\title{
El movimiento cooperativo argentino y sus organizaciones: trayectoria y perspectivas
}

\author{
Mirta Vuotto \\ Profesora consulta. Directora del Centro de Estudios de Sociología del Trabajo. \\ Universidad de Buenos Aires, Facultad de Ciencias Económicas \\ mirta.vuotto@gmail.com
}

Sumario: Sumario: 1. Introducción.-2. El contexto de origen.-3. La evolución desde el siglo xx.-4. Tendencias y problemáticas a inicios del siglo XXI. - 4.1. La estructura y posicionamiento del sector.—4.2. El Estado y el movimiento cooperativo.- 5 . Conclusiones.-Referencias.

Resumen: El propósito del artículo es caracterizar la situación actual del cooperativismo argentino, en términos de su contribución económica y social, sus potencialidades y limitaciones. El texto está estructurado en tres secciones. En la primera se realiza una breve referencia al desarrollo del movimiento desde su origen, para considerar en la segunda sección los procesos de desarrollo durante el siglo xx y comienzos del siglo actual. Se considera en este ámbito el posicionamiento de las entidades según el interés de su membrecía, el sector de actividad al que pertenecen y los puestos de trabajo generados. En la tercera sección se analiza el papel del Estado y las políticas públicas orientadas al sector. Sobre estas referencias se presentan las principales conclusiones del análisis.

Palabras clave: cooperativas, membrecía cooperativa, empresas cooperativas.

Abstract: The aim of this article is to clarify the situation of Argentine cooperatives, in terms of their economic and social contribution, their potentialities and limitations. The article is structured in three different sections. In the first one a brief reference is made to the development of the movement from its origin. The second section is devoted to describe the development processes during the 20th century and the beginning of the 21st. The positioning of the entities is considered according to the interest of their membership, the activity sector, and the jobs generated. The third section analyzes the role of the State and the public policies oriented to the sector. The main conclusions of the analysis are presented on these references.

Keywords: cooperatives, cooperative membership, cooperative enterprises. 


\section{Introducción}

En el artículo se analiza la trayectoria y perspectivas del movimiento cooperativo y sus organizaciones con el propósito de caracterizar las principales contribuciones. Se considera en el análisis su posicionamiento y problemáticas en el contexto de una sociedad que refleja desde hace décadas una profunda y persistente desigualdad social, altos niveles de pobreza - agravados por el estancamiento de la economía desde hace once años- y limitaciones de la dirigencia política para encontrar un sendero de desarrollo. A estos factores se añade el impacto del COVID-19 al poner al descubierto el alcance de una crisis que se expresa en los planos sanitario, económico y social, así como en la concentración de poder político y el control al que se recurre para enfrentarla.

La metodología empleada para analizar el desarrollo y las problemáticas del movimiento en distintas coyunturas está basada en fuentes primarias provenientes de las organizaciones cooperativas, publicaciones sobre la temática, y entrevistas a interlocutores competentes en las entidades representativas del movimiento.

El artículo se estructura en tres secciones. En la primera se considera el origen del movimiento, para describir en la segunda sección los procesos de desarrollo desde el siglo Xx y el posicionamiento de las cooperativas según el interés de su membrecía, la actividad económica y los puestos de trabajo generados. En el contexto de los ciclos de inestabilidad socioeconómica de las últimas décadas se ilustra en la tercera sección el carácter de las políticas públicas orientadas al sector y los desafíos que le plantean al movimiento. Finalmente, en las conclusiones, se retoman las principales evidencias del estudio en función de los objetivos del artículo.

\section{El contexto de origen}

En los años posteriores a la conformación de la Argentina como estado independiente, en 1810, se produjeron importantes transformaciones políticas, sociales, económicas y culturales que cambiaron la fisonomía de su sociedad. Junto con los cambios sustanciales en el orden político, el país comenzó a insertarse a mediados del siglo XIX en una economía mundial caracterizada por el acelerado crecimiento de la producción y la creciente integración de los mercados, para conformar una Argentina moderna a partir de las dos últimas décadas del siglo xIx. Sus rasgos distintivos fueron la consolidación de un Estado cen- 
tral, la afirmación de una economía capitalista y la formación de una sociedad dinámica y compleja.

Se ha destacado en ese contexto la importancia del surgimiento de organizaciones y prácticas asociativas entre sectores muy diversos, tanto social como culturalmente e iniciativas de ayuda mutua, promovidas por pequeños productores rurales y por trabajadores urbanos. En ellas, los inmigrantes europeos desempeñaron un papel protagónico debido a su experiencia anterior en los países de origen, por lo que realizaron un significativo aporte a la formación de las asociaciones cooperativas, mutualidades y sindicatos.

La consolidación del Estado nacional, basada en el predominio del litoral atlántico sobre el interior del país, se afianzó en un modelo de expansión y desarrollo sobre tres pilares: la explotación agropecuaria, las inversiones extranjeras ${ }^{1}$, la inmigración y la educación universal y obligatoria, que actuó hasta mediados del siglo xx como principal canal de movilidad social.

En el plano social, a partir de 1880, las élites liberales que dirigieron la organización del país impulsaron un activo proceso inmigratorio² con el objeto de sustituir la vieja estructura heredada de la sociedad colonial, por otra inspirada en los modelos de los países más avanzados de occidente (Germani, 1962). Como resultado de la aceleración de un proceso inmigratorio que adquirió un carácter masivo ${ }^{3}$, la población se multiplicó casi seis veces entre 1860 y 1900. La masividad del proceso implicó una renovación sustancial de la población del país respecto de la población nativa residente, en particular en las zonas de mayor importancia económica, social y política.

1 Las inversiones extranjeras, particularmente de origen inglés financiaron entre un tercio y la mitad de la inversión física neta del país, alcanzando en 1913 su punto máximo. Cerca del 50\% del capital fijo existente era propiedad extranjera y el $75 \%$ estaba destinado a proporcionar el capital básico de infraestructura en transporte y servicios públicos. Así, las inversiones extranjeras financiaron la inversión y el gasto público a través de la absorción de títulos de gobierno. La magnitud del endeudamiento externo determinaba que los servicios de la deuda representaran una proporción muy elevada de las divisas generadas por las exportaciones argentinas (Ferrer,1968).

2 Este proceso fue promovido por la Ley 817 de 1876. La inmigración de ultramar ( $50 \%$ italianos y $30 \%$ españoles seguido de polacos rusos, franceses y alemanes) se concentró en los territorios más aptos para la agricultura intensiva y en los principales centros urbanos para conformar un heterogéneo mosaico de trabajadores que configuró el principal estrato de la clase trabajadora y fue la base del gran desarrollo urbano.

3 La Argentina era uno de los países menos poblados de América del Sur y según el primer Censo de población de 1869 contaba con menos de 1.800 .000 habitantes. El $80 \%$ de esa población eran analfabetos; más del $70 \%$ de las viviendas eran ranchos de paja y de cada 100 niños en edad escolar sólo 20 iban a la escuela. 
La mayoría de los inmigrantes, agricultores de origen, fueron atraídos por la promesa de distribución de tierras, aunque la ausencia de un programa nacional de colonización impidió que el campo absorbiera las corrientes migratorias provenientes del exterior. Al cercenarse el desarrollo de una producción agropecuaria con base en productores medios se conformó una estructura agraria polarizada, que se caracterizó por una parte sustancial de la superficie disponible explotada por arrendatarios y otra parte significativa conformada por grandes unidades productivas. Numerosos inmigrantes se establecieron en las áreas urbanas como obreros, artesanos y comerciantes, aumentando la oferta de mano de obra disponible, con su consiguiente efecto depresivo sobre el nivel de salarios y el crecimiento de la desocupación urbana.

Hasta 1930, el sindicalismo, la acción socialista parlamentaria y la protesta anarquista, entre otras, fueron expresiones de una sociedad civil que reclamaba cambios institucionales y mayor participación en los asuntos públicos. Asimismo, en la búsqueda de mejores condiciones de vida, los migrantes desarrollaron experiencias de características cooperativas, algunas veces fallidas, en el sector urbano ${ }^{4}$, con una notoria influencia del cooperativismo europeo e impulsadas por la misma lógica asociativa que estimuló a las organizaciones de base y los sindicatos.

El funcionamiento de las cooperativas que se desarrollaron durante el siglo XIX en Europa fue conocido en la Argentina, principalmente a través de las ideas expuestas y la actividad desplegada por la iniciativa de numerosos inmigrantes europeos. También se experimentaron naturalmente algunas experiencias fallidas que caracterizaron las primeras épocas del movimiento cooperativo. Al respecto se menciona la carencia de conocimientos adecuados acerca de la verdadera forma y principios de las entidades cooperativas, la actuación dentro de un medio indiferente $u$ hostil y la ausencia de una legislación apropiada que permitiera diferenciar netamente a las cooperativas de otros tipos de entidades y sancionara el uso indebido de la denominación cooperativa. (Kaplan de Drimer; Drimer 2017, pp. 585-586).

4 Entre las primeras experiencias se encuentra la «Sociedad Cooperativa de Producción y Consumos» creada en 1875 e inspirada por el ciudadano francés Adolfo Vaillant, la cual no alcanzó a funcionar, y la "Sociedad Cooperativa de Almacenes» fundada en 1884 por el británico Carlos Atwell, considerada la primera cooperativa con sede en Buenos Aires y dirigida al consumo de comestibles y bebidas. Se basó en los sistemas ya difundidos en Inglaterra y funcionó como una cooperativa de empleados. Desapareció en 1890 con motivo de la gran crisis económica que vivió el país (Kaplan de Drimer; Drimer, 2017). 
En ese contexto, entre las numerosas experiencias cooperativas conformadas durante el siglo XIX y el primer tercio del siglo XX algunas han sido las primeras en su género dentro del país y otras lograron alcanzar un notable desarrollo. La coexistencia en la primera etapa de iniciativas relevantes, con otras que se denominaban cooperativas, aunque sin respetar en su funcionamiento los principios y valores, condujo a que la difusión y observancia de los principios cooperativos fuese una preocupación temprana del movimiento y que la integración entre cooperativas constituyese un camino para su desarrollo (Caletti, 1988).

Entre las cooperativas creadas a fines de la década de 1890, dos de ellas se destacaron por conservar el carácter genuino de sus principios: «El Progreso Agrícola de Pigüe», fundada por inmigrantes franceses para cubrir los riesgos derivados de las inclemencias del tiempo, que contó con el asesoramiento de Charles Gide para la redacción de sus estatutos (Fernández, 1916) y la "Cooperativa Obrera de Consumos» creada por el dirigente socialista Juan B. Justo e inspirada en los principios de Rochdale. En su estatuto de quince artículos adoptó normas tales como el acceso libre, la adhesión voluntaria, la organización democrática y las ventas al contado; también aplicó un método cooperativo de distribución de los excedentes, para destinar el $60 \%$ a los asociados en proporción a su consumo, el 30\% al fondo de reserva y el 10\% a los empleados (Kaplan de Drimer; Drimer 2017, p. 589-590).

Basándose en las experiencias precedentes, el impulso del cooperativismo bajo la influencia de la inmigración permite resaltar el papel precursor de los pequeños productores rurales en la creación de cooperativas agrarias de comercialización y de los grupos de obreros industriales y artesanos que fomentaron en especial el cooperativismo de consumo, al compás del notable crecimiento urbano, especialmente en Buenos Aires, Rosario y en otras ciudades del litoral argentino. En el censo de 1914 se registraban 40 sociedades cooperativas, de las que 21 se localizaban en la Ciudad de Buenos Aires y 19 en el interior. En conjunto contaban con 27.661 asociados, lo que permitía afirmar que

el espíritu mutualista y cooperativo está profundamente arraigado [...] debido a que la inmensa mayoría tiene la clara visión de los evidentísimos perjuicios morales económicos y sociales que ocasionan los intermediarios en muchísimas operaciones de la vida urbana y en todas las operaciones agrícolas, así como de los que causan los explotadores vulgares de la producción del trabajo, del crédito, del consumo y de la previsión.» (Bórea, 1917, p. 227)

Varios autores destacaron de importancia en esta etapa la autonomía en el surgimiento de las cooperativas, como su estructura en torno 
a los principios de Rochdale y su conformación como entidades al servicio de las necesidades de sus asociados.

\section{La evolución desde el siglo xx}

Los ciclos de auge del cooperativismo durante el siglo xx coincidieron con la creación de nuevas entidades en algunas ramas, la consolidación de otras en ámbitos específicos de la actividad económica y el contraste de las dinámicas evolutivas en consonancia con los cambios socioeconómicos y políticos del país. La evolución, asociada a las condiciones del desarrollo regional coincidió con el notorio auge de las cooperativas agrarias en las décadas de 1940 y 1950 e impulsó las iniciativas de integración que se concretaron a través de la creación de entidades de segundo grado como la Asociación de Cooperativas Zona Central fundada en 1922. En esta perspectiva, los procesos de intercooperación permitieron conformar organismos de integración que de distinto modo han servido para defender, representar y promover el desarrollo de las cooperativas 5 . Del mismo, modo, las entidades constituidas han podido brindar a las cooperativas de base herramientas para su desarrollo, y actuaron además como representantes políticos de estas organizaciones ante los gobiernos, los medios y la sociedad civil, promoviendo la fórmula asociativa y los valores de la cooperación. La organización de tercer grado de índole nacional que agrupa al sector cooperativo agrario es la Confederación Intercooperativa Agropecuaria Limitada (Coninagro), fundada el 18 de septiembre de 1956. La entidad, fue creada con el propósito de orientar los procesos de desarrollo del movimiento y unificar acciones de defensa y representación nacional e internacional.

Asimismo, se afirmó el papel de las entidades de servicios públicos -impulsoras del desarrollo en centros poblados pequeños y medianos (Kaplan de Drimer; Drimer, 1917. CEPAL, 1989) - a partir de la importancia desde 1926 de las cooperativas del sector eléctrico, conformadas como actores primordiales de la distribución de energía en el interior del país y constituidas en líderes en el ámbito de la electrificación rural.

${ }^{5}$ El primer organismo fue la Confederación Entrerriana de Cooperativas, originado en 1913 en ocasión de celebrarse el Primer Congreso Provincial de Cooperativas, convocado con la finalidad de que las cooperativas pudiesen confrontar sus estatutos sociales, intercambiar experiencias y organizar actividades comerciales conjuntas. La confederación que surgió del congreso tuvo una vida efímera, aunque fue refundada con el actual nombre de Federación Entrerriana de Cooperativas. 
De forma paralela, la rama del cooperativismo de seguros amplió su influencia hasta convertirse en el sector con mayor cantidad de asociados del cooperativismo nacional. Parte del éxito se debió a su accionar en los ámbitos rurales y su eficacia para contemplar y dar respuesta a los eventos climáticos que estaban excluidos de la cobertura usual de las empresas de seguro convencionales. El desarrollo de las cooperativas urbanas en el campo del consumo, ahorro y crédito, producción y vivienda y la necesidad de integración condujeron en 1962 a la creación de la Confederación Cooperativa de la República Argentina (actualmente Cooperar) mientras que el cooperativismo agrario hasta 1980 continuó manteniendo el número de entidades e incrementó el número de asociados.

En la alternancia de sucesivos ciclos de inestabilidad política que condicionaron el afianzamiento y el desarrollo posterior cabe mencionar el cambio producido a partir del restablecimiento de la democracia durante el período 1984-1988. En esa coyuntura el movimiento cooperativo fue visualizado desde el Estado como una herramienta adecuada para el fortalecimiento de la sociedad civil, dado su estructura participativa y su poder democratizador. Por ello fue reconocido como un eficaz instrumento de organización socioeconómica, con capacidad para defender los intereses de sus integrantes e impulsar el desarrollo social, destacándose por su profunda voluntad de transformar las instituciones. Durante ese período se produjo un cambio significativo en cuanto al surgimiento de entidades y se revirtió la tendencia declinante iniciada al comienzo del proceso militar del año 1976 (Levín; Verbeke,1997).

Hacia fines de 1983 dejó de publicarse la síntesis estadística oficial sobre las sociedades cooperativas, de modo que la información sobre las tendencias en la evolución de cada rama se pudo constatar hacia fines de la década de 1990, con base en los datos de un reempadronamiento general realizado por la autoridad de aplicación. El mismo puso en evidencia la significativa desaparición de entidades y asociados en el movimiento (INACyM, 1999).

\section{Tendencias y problemáticas a inicios del siglo XXI}

La crisis económica argentina que estalló a fines de 2001, luego de un periodo de recesión que se extendió durante tres años, se vio reflejada en los altos niveles de pauperización de una parte significativa de la población, en la reestructuración del mercado de trabajo, la declinación en la tasa de crecimiento del producto y la caída generalizada de ingresos. El agravamiento de la situación social se manifestó en niveles 
inusitados de pobreza y desigualdad. En ese contexto, las reformas institucionales impusieron una visión estrecha de la política social al postular como objetivo casi excluyente atender las situaciones de pobreza extrema.

El cooperativismo fue particularmente afectado por esa situación y experimentó distinto tipo de transformaciones y cambios en el posicionamiento de algunas ramas. Por un lado, las cooperativas de trabajo resultaron dominantes debido a un notable crecimiento desde la década de 1990 y por otro, las cooperativas agropecuarias, de seguros y vivienda, y en menor grado las de consumo, crédito y provisión fueron los sectores más afectados por la reducción de las entidades activas y en algunos casos por su desaparición ${ }^{6}$. Como resultante, del total de 16.059 cooperativas activas en 2001 el 42\% eran cooperativas de trabajo, $19 \%$ de vivienda y construcción, $14 \%$ agropecuarias, $12 \%$ de servicios públicos, $10 \%$ de provisión y el resto de crédito y consumo.

La configuración de un nuevo horizonte político luego de la crisis posibilitó el cambio de orientación en la política social, coincidente con el interés por incorporar diversas estrategias de reducción de la pobreza a través de programas de transferencia de ingresos a la población más pobre. Una vez recuperado el crecimiento económico, la mejora de los indicadores sociales posibilitó pasar de dichos programas a la implementación de estrategias de protección social basadas en la creación de puestos de trabajo. De esta forma la problemática de la exclusión económica se incorporó en primer término a la agenda política y luego a la agenda institucional en el marco de una intervención del Estado orientada a la economía social y al estímulo de las cooperativas.

Desde el punto de vista de su impacto, esas intervenciones se realizaron en el marco de políticas sectoriales y territoriales que reconocieron el papel social de las cooperativas y su contribución en materia de empleo y a través de instrumentos de alcance general, con uno o varios caracteres comunes. Las políticas abarcaron numerosas iniciativas asociativas sin diferenciar el sector de actividad y el territorio de pertenencia y en el diseño de los programas como en su implementación,

6 Cabe mencionar el impacto de algunos procesos, particularmente en el sector de crédito, a partir de la vigencia de La Ley 21.526 de Entidades Financieras y el claro sentido restrictivo sobre la operatoria de las cajas de crédito y los bancos cooperativos. Así, a inicios de la década de 1970, de las 974 cooperativas de crédito que operaban en más de trescientas localidades de 17 provincias y manejaban el 10,5\% del total de los depósitos del sistema financiero se mantuvieron activas hacia fines de 1972 solo 34 entidades. Asimismo, los 85 bancos cooperativos que funcionaban en 1979 quedaron reducidos a dos entidades en 2000, de las que solo perdura una. 
el Ministerio de Desarrollo Social (MDS) jugó un papel protagónico al impulsar planes y proyectos orientados a la creación y fortalecimiento de emprendimientos socioproductivos, mutualistas y cooperativos. Las políticas sociales plantearon la necesidad de establecer condiciones institucionales adecuadas para la promoción, organización y apoyo al desarrollo local en una perspectiva estratégica de alcance regional y nacional que incluyese diversas formas asociativas. Entre los instrumentos implementados por el MDS los más relevantes fueron el Plan Nacional de Desarrollo Local y Economía Social «Manos a la Obra» ${ }^{7}$, tendiente a mejorar la calidad de vida de las familias y generar condiciones favorables para la integración social a través del sistema productivo y el Programa de Ingreso Social con Trabajo, (Resolución MDS n. ${ }^{\circ} 3182 / 2009$ ) instituido en agosto de 2009 con el propósito de estimular oportunidades genuinas de empleo en cooperativas, por considerar al trabajo «como el mejor antídoto contra la pobreza». Hacia fines de 2019 estaban matriculadas más de veintisiete mil cooperativas de trabajo de las que formaban parte 427 empresas recuperadas que empleaban más de 16.000 trabajadores (INAES, 2021). De ese total permanecen 8.140 cooperativas de trabajo activas y solo 2796 de las que fueron creadas en el marco de los programas y en distinto grado se encuentran afectadas por las restricciones durante la pandemia. En el contexto de una nueva institucionalidad pública numerosos programas mantuvieron continuidad y se añadieron mayores recursos y nuevos instrumentos en particular con el propósito de recuperación del empleo en los sectores más afectados.

\subsection{La estructura y posicionamiento del sector}

De acuerdo con un estudio sobre las fuentes estadísticas del cooperativismo argentino (Vuotto, Estragó, 2021) se puede caracterizar la estructura del sector con base en la fuente disponible en el INAES y sistematizar los datos provenientes del padrón estructurado sobre los registros que proveen al organismo las cooperativas matriculadas. Sobre la cobertura de esa fuente oficial, a partir de la actividad económica principal de las cooperativas, la cantidad de asociados y el empleo generado, se pueden conformar tres campos diferenciados: el de coope-

7 En 2004 comenzó a delinearse el marco normativo que permitiría su puesta en funcionamiento y se dictó la resolución de creación del programa No 1375/04. La autoridad de aplicación (art.9) fue la Secretaria de Políticas Sociales. La Subsecretaría de Desarrollo Territorial y Economía Social fue responsable de la gestión y coordinación. 
rativas de productores, de trabajadores y de consumidores/usuarios. La configuración resulta de la clasificación propuesta por la OIT aunque en el caso argentino integran ese conjunto las cooperativas de múltiples partes interesadas o modalidad en la que miembros de diversa naturaleza (trabajadores, consumidores y/o productores) comparten la representación en los órganos societarios, sin posición dominante de un grupo de ellos. En la resolución 478/2020 el INAES establece la posibilidad de constituir entidades cooperativas de múltiples partes interesadas definiéndolas como integradas «con asociados que cumplen diferentes roles en la cadena de producción y consumo, vinculados entre sí por la proximidad geográfica» en las que hasta un tercio de sus asociados son personas de otro carácter jurídico. Esta resolución refiere explícitamente a entidades de grado superior.

A partir de la clasificación precedente se muestran en las columnas $a, b, y$ c de la tabla 1 tres tipos de entidades según las dimensiones mencionadas. En la columna d se presenta el agrupamiento con las modalidades desagregadas para cada tipo.

Tabla 1

\section{Cooperativas matriculadas: número de entidades, asociados y puestos de trabajo en Argentina (año 2021)}

\begin{tabular}{|c|c|c|c|c|c|c|}
\hline $\begin{array}{l}\text { Tipo de } \\
\text { cooperativa } \\
\text { (a) }\end{array}$ & $\begin{array}{l}\text { Interés } \\
\text { membrecía } \\
\text { (b) }\end{array}$ & $\begin{array}{l}\text { Tipo de } \\
\text { miembros } \\
\text { (c) }\end{array}$ & $\begin{array}{l}\text { Clasificación } \\
\text { según INAES } \\
\text { (d) }\end{array}$ & $\begin{array}{c}\text { Entidades } \\
\text { (e) }\end{array}$ & $\begin{array}{l}\text { Asociados } \\
\text { (f) }\end{array}$ & $\begin{array}{l}\text { Puestos } \\
\text { de trabajo } \\
\text { (g) }\end{array}$ \\
\hline \multirow{2}{*}{$\begin{array}{c}\text { De } \\
\text { productores }\end{array}$} & \multirow{2}{*}{$\begin{array}{l}\text { Actividad } \\
\text { productiva }\end{array}$} & \multirow{2}{*}{ Productores } & Agropecuaria & 718 & 219.030 & 18.726 \\
\hline & & & Provisión & 620 & 368.464 & 6.901 \\
\hline $\begin{array}{c}\text { De } \\
\text { trabajadores }\end{array}$ & Trabajo & Trabajadores & $\begin{array}{l}\text { Trabajo y } \\
\text { trabajadores }\end{array}$ & 8.140 & 181.398 & 186.460 \\
\hline \multirow{5}{*}{$\begin{array}{c}\text { De } \\
\text { consumidores / } \\
\text { usuarios }\end{array}$} & \multirow{5}{*}{ Consumo } & \multirow{5}{*}{ Consumidores } & Consumo & 129 & 1.951 .992 & 4.088 \\
\hline & & & $\begin{array}{l}\text { Vivienda y } \\
\text { construcción }\end{array}$ & 738 & 1.251 .079 & 4.418 \\
\hline & & & $\begin{array}{l}\text { Servicios } \\
\text { públicos }\end{array}$ & 1.178 & 4.076 .800 & 25.048 \\
\hline & & & Crédito & 142 & 2.128 .805 & 6.000 \\
\hline & & & Seguros & 18 & 8.327 .991 & 5.797 \\
\hline \multicolumn{4}{|c|}{ TOTALES } & 11.683 & 18.505 .559 & 257.438 \\
\hline
\end{tabular}

Fuente: elaboración propia basada en el registro de entidades INAES 2021. 
Los datos corresponden a las entidades clasificadas por el INAES como "vigentes», es decir se excluyen aquellas que en el padrón se presentaban en la categoría cese de actividades (suspendida, cancelada o con retiro de autorización para funcionar), así como las entidades que no informaban el número de asociados.

Tampoco se tuvo en cuenta a las cooperativas de grado superior (federaciones y confederaciones). Las columnas e, f y g indican, respectivamente, el número de entidades vigentes, asociados-usuarios y puestos de trabajo generados según el agrupamiento correspondiente.

En el perfil sectorial del cooperativismo argentino que conforman los indicadores de la tabla 1, a partir del número de entidades se destaca la categoría de cooperativas de trabajadores, por constituir la notoria mayoría, con cerca del $70 \%$ de las matriculaciones. En este significativo perímetro, un heterogéneo conjunto de entidades está representado en gran medida por cooperativas inducidas por el Estado. La creación «desde arriba» implementada por las políticas públicas ha transitado entre los extremos que la propia naturaleza de estas organizaciones lo permite. Prevalecen actitudes reduccionistas al ser visualizadas como solución de emergencia ante las crisis, junto con posturas de cooptación, cuando el Estado intenta jugar un rol económico activo y dominante al impulsar su creación. En este caso, las políticas de promoción equivalen a una tutela que restringe las iniciativas autónomas y que pierde de vista que la cooperativa evoluciona según un ciclo de vida que requiere en muchos casos del acompañamiento, debido a que en su trayectoria van cambiando sus características y requerimientos.

Con apreciable distancia de la categoría anterior se ubican las cooperativas de consumidores-usuarios y las de productores. En las primeras - de usuarios - cabe destacar la importancia que mantienen las cooperativas de servicios públicos, en particular las cooperativas de electricidad que, desde su origen en la segunda década del siglo $x \mathrm{x}, \mathrm{y}$ posteriormente, se fueron creando sobre las mismas bases, con el propósito de defender a sus asociados frente a las condiciones abusivas impuestas por las compañías de electricidad privadas oferentes del servicio. Dichas compañías habían reemplazado a las empresas nacionales existentes y se vinculaban con grupos de origen extranjero (Kaplan y Drimer, 2017, pp. 599-600). La prestación del servicio de electricidad constituyó el objeto principal de estas cooperativas, con una cobertura extendida al $80 \%$ de las redes de electrificación rural en el país. Progresivamente brindaron otros servicios esenciales como gas, agua potable, cloacas, telecomunicaciones etc.

Las cooperativas de servicios públicos están sujetas a diversos organismos de fiscalización y desde hace décadas han sido afectadas por 
la ausencia de marcos normativos adecuados y por limitaciones en la coordinación de sus objetivos con los organismos públicos regulatorios. La problemática reconocida por el sector radica en un marco regulatorio que, al tener vigencia para concesionarios comerciales, desvirtúa la naturaleza cooperativa de una empresa propiedad de sus usuarios y tiende a disociar cooperativa y asociado por considerar a las entidades como concesionarias y convertir al asociado en mero usuario/cliente.

En el contexto del retail y el consumo masivo, el cooperativismo de consumo afirmó desde fines de 2000 su presencia en el país, en particular con una de las entidades centenarias constituida en la provincia de Buenos Aires que cuenta con sucursales en cuatro provincias y representa el $91 \%$ de los asociados al sector y el 3,9\% del mercado local. La cooperativa ofrece entre otras, más de mil cien referencias a través de siete marcas propias (Balance social cooperativo, 2019). En los últimos años el conjunto de cooperativas de la rama se ha visto afectado por la economía informal, la volatilidad de costos y los nuevos patrones de comportamiento e interacción de los asociados con la cooperativa y con las marcas, como consecuencia del distanciamiento social resultante de la pandemia.

En cuanto al tema del hábitat, no obstante un importante desarrollo de la construcción residencial luego de la crisis de 2001, cabe señalar que las viviendas construidas no se ajustaron a las dinámicas poblacionales. Por esta razón, la demanda originada en el crecimiento vegetativo de la población, que alcanza el $1 \%$, plantea la necesidad de disponer de 200 mil soluciones al año para poder comenzar a impactar en alguna medida en reducir el déficit existente (Secretaría de Vivienda, 2019). En ese contexto resulta significativo mencionar que en siete provincias las cooperativas promueven procesos de organización colectiva de esfuerzo propio, ayuda mutua y autogestión del hábitat. En el ámbito de la Ciudad de Buenos Aires se destaca el Programa de Autogestión de la Vivienda en el que un amplio conjunto de organizaciones, movimientos, partidos políticos y numerosas familias ocupantes e inquilinas, constituyeron un escenario multiforme y diverso de 534 cooperativas (Pedro et al., 2021].

Finalmente, en el ámbito de las organizaciones de consumidoresusuarios cabe destacar la representatividad de dieciocho entidades que ofrecen cobertura de riesgos asegurables de distinta naturaleza. Dos de ellas integran el ránking de aseguradoras líderes ${ }^{8}$ en atributos como vi-

8 Una de ellas concentra el 30,7\% del mercado de los seguros agropecuarios y forestales, mientras que otra lidera el mercado de riesgos del trabajo y tiene en ese rubro el 8,9\% de la participación en el negocio (Todo riesgos, 2021), 
sión sustentable y cumplimiento de su compromiso con los asegurados. Todas se encuentran afectadas en distinto grado por la inflación, al incidir en la sensible disminución de sus fondos e impedir el resguardo adecuado de los pagos que deben afrontar. A ello se agregan las restricciones para realizar inversiones en bonos $u$ otros activos en moneda extranjera y las devaluaciones del peso con su alto impacto en los programas de reaseguro en moneda extranjera.

El grupo más reducido de entidades - cooperativas de productores - se integra en partes similares por las cooperativas agropecuarias y las de provisión. Las primeras atravesaron un profundo proceso de transformación institucional, que se inició en la década de 1970, y se aceleró generalizándose en el marco de las políticas de apertura y desregulación de la década de 1990. Aunque los fuertes procesos de adaptación condujeron a la desaparición de numerosas organizaciones en el medio rural, un conjunto significativo mantiene su participación en los distintos mercados de productos agropecuarios, incluidos los tradicionales. La actual coyuntura expresa para estas cooperativas una situación compleja debido a ciertos aspectos que se vinculan con las producciones de las economías regionales: una política cambiaria poco transparente, que incide en la compra y venta de insumos, y el tema inflacionario. La incertidumbre de las políticas económicas de mediano y largo plazo generan un marco de inestabilidad que perjudica en particular las inversiones en el sector.

En cuanto a las cooperativas de provisión se incluyen los casos de entidades que ofrecen servicios para la producción, en particular para productores y emprendedores de diversos rubros (agrícola, transporte, farmacéutico, comercio minorista en ferias, etc.).

En segundo lugar, desde la perspectiva del número de asociadosusuarios, la caracterización anterior se modifica sensiblemente dado que prevalece en el conjunto un cooperativismo de consumidores. Es importante señalar la diversidad de situaciones de una membrecía cooperativa asociada a múltiples entidades: un productor agropecuario puede estar asociado al mismo tiempo a una cooperativa agrícola, una de servicios públicos, una de provisión, una de seguros, una de crédito y una de consumo. Adicionalmente, los datos disponibles permiten apreciar que las redundancias se suceden inclusive dentro de un mismo sector. A título de ejemplo, se destaca el caso de la provincia de Santa $\mathrm{Fe}$, que cuenta con casi 4,9 millones de asociados a cooperativas de seguros, cuando la población estimada de la provincia es de alrededor de 3,4 millones de habitantes. En este caso dos entidades de seguros informan el número de asociados de todo el país a través de la sede localizada en esa provincia. Aunque la frecuente duplicidad de registros 
limita la perspectiva de clasificación para el total, según los asociados, permite - al menos - entrever dónde se focaliza la mayor «densidad de actividades cooperativas». Resulta destacable la posición dominante que revelan las cooperativas de seguros, las de servicios públicos y -en menor medida_ las entidades dedicadas al crédito y al consumo.

En la caracterización del perfil del cooperativismo argentino según los puestos de trabajo se pone de manifiesto nuevamente el protagonismo de las cooperativas de trabajadores. La cantidad de asociadostrabajadores en este tipo de entidades, equivale a casi tres de cada cuatro empleos cooperativos. Cabe destacar que estos empleos se diferencian del resto debido a que no se trata de trabajadores en relación de dependencia. En este ámbito, es importante subrayar la naturaleza jurídica del vínculo que liga a los trabajadores con las cooperativas en las que se desempeñan ya que, en estos empleos, se identifican en muchos casos organización e ingreso regular periódico, con relación de dependencia sin admitir la posibilidad de una organización del trabajo que no sea en relación de dependencia (Cracogna, 2016, p. 62). El vínculo asociativo, propio de la organización, configura la realización de actos esencialmente cooperativos, previstos por el artículo 4 de la Ley 20.337 ya que este tema fundamental influye en forma decisiva sobre casi todos los demás aspectos relacionados con el trabajo autogestionado (Vuotto, 2011). En conjunto, los 262.329 puestos de trabajo de las cooperativas representan apenas el 1,5\% del empleo del país, cifra que alcanza una mayor representatividad en contextos en que la presencia cooperativa constituye un factor dinamizador de la economía local (empleo en sector agropecuario y en los servicios públicos).

En cuanto a la localización de las cooperativas vigentes sobresale la mayor concentración en la región Pampeana. Al respecto, la producción argentina se concentra principalmente en esa región y de manera específica, la provincia de Buenos Aires y la Ciudad Autónoma de Buenos Aires generan más de la mitad del producto bruto del país. En consonancia con esos datos se produce la mayor concentración de cooperativas, en particular las del sector agropecuario e industrial. La localización también se corresponde con el empleo privado registrado, cuya distribución regional es similar al de las cooperativas en el ámbito territorial.

En la mayor parte de los casos el papel de las cooperativas en cada ámbito -y su colaboración con otras entidades, la membrecía y otras partes interesadas - puede ayudar a conformar una red global de actores que operan a nivel local. Sus valores y principios les permiten constituirse en actores proactivos en la implementación de procesos de desarrollo sostenible de una manera eficiente, democrática y centrada en las personas. 


\subsection{El Estado y el movimiento cooperativo}

Las relaciones entre el Estado y el movimiento cooperativo se instituyen en planos diferentes. Por un lado, en lo referido al cumplimiento de la función de supervisión, control y fomento que debe ejercer el Estado en resguardo de los intereses de la sociedad en general, y por otro en cuanto a la representación en el Estado de la membrecía organizada en cooperativas.

En el primer plano, el Instituto Nacional de Asociativismo y Economía Social (INAES), en el ámbito del Ministerio de Desarrollo Productivo constituye la autoridad de aplicación del régimen que regula el funcionamiento de las cooperativas, así como su promoción ${ }^{9}$. Sus competencias se han establecido en la Ley 20.337, sus modificatorias y complementarias y las normas dictadas al respecto ${ }^{10}$.

En el segundo plano, de acuerdo con el artículo 4 del Decreto n. ${ }^{\circ} 420 / 96$, la conducción y administración del INAES se integra en su directorio por un presidente y seis vocales de los cuales dos son designados en representación de las cooperativas. Las distintas entidades que agrupan a las cooperativas propiciaron en 2019 la designación como sus representantes de los presidentes de Cooperar y Coninagro.

En función de las orientaciones y objetivos que privilegian los distintos organismos gubernamentales —en un ámbito específico de la sociedad o en un territorio- se pueden identificar y delimitar distintas políticas según su carácter horizontal o vertical-sectorial (Kendall, 2001). Al respecto se diferencian las políticas horizontales, dirigidas al conjunto

9 El INAES (Decreto n. ${ }^{\circ} 721 / 2000$ ) ejerce en el ámbito nacional las funciones que le competen al Estado Nacional como autoridad de aplicación del régimen legal que regula el funcionamiento de las Asociaciones Mutuales y las Cooperativas establecido por las Leyes 20.321 y 20.337. Tiene a su cargo el otorgamiento de la personería jurídica a las entidades a través del registro nacional de cooperativas y mutuales, ejerce, en el mismo ámbito, su control público y superintendencia Fomenta el desarrollo, educación y promoción de la acción cooperativa y mutual en el país, el reconocimiento de estas organizaciones efectuando el otorgamiento, denegatoria o retiro de la personería jurídica para su funcionamiento, como así también su superintendencia y control público. Para el cumplimiento de sus misiones y funciones el organismo cuenta con financiamiento propio otorgado por la Ley 23.427 y los derivados del aporte contemplado en el artículo 9 de la Ley 20.321.

10 Existen además organismos provinciales con atribuciones de distinta naturaleza en lo relativo a la organización y aplicación de la normativa nacional en las actividades de las cooperativas en sus respectivas jurisdicciones. Esos organismos tienen diferente status jerárquico (direcciones generales de inspección de personas jurídicas o inspecciones generales que por lo general llevan un registro provincial de organizaciones de la comunidad) y dependen en algunos casos de los ministerios de justicia de la provincia o de fiscalías de Estado. 
de organizaciones de la economía social, de las verticales que incluyen a subcampos determinados, que a la vez pueden contener grandes conjuntos o subsectores (instituciones sin fines de lucro o empresas autogestionadas) o algunas de sus formas institucionales (cooperativas, asociaciones, etc.). La sectorialidad de las políticas se concibe en el sentido de ramas de actividad económica como la agraria, la industrial o la de servicios, y con las políticas estructurales horizontales, como desarrollo regional y local, por ejemplo.

En el ámbito de las políticas públicas, el conjunto programas y categorías programáticas dirigidas al cooperativismo se muestra en la tabla 2 donde se agrupan los programas e instrumentos vigentes, cuya sectorialidad está determinada a partir de los ámbitos de competencia de las instituciones responsables y los distintos sectores de actividad económica. Mientras que los programas están integrados por un conjunto de proyectos que persiguen los mismos objetivos y establecen las prioridades de la intervención en términos de la asignación de los recursos, los proyectos constituyen la unidad mínima de asignación de recursos para el logro de uno o más objetivos específicos y de forma agregada, constituyen el punto de partida más adecuado para formular apreciaciones sobre los programas.

Tabla 2

\section{Políticas públicas orientadas a las cooperativas: categorías programáticas incluidas en el presupuesto de la Administración Nacional (2020-2021)}

\begin{tabular}{|c|c|}
\hline $\begin{array}{l}\text { Instituciones } \\
\text { responsables }\end{array}$ & Categorías programáticas \\
\hline & Promoción de empleo social \\
\hline $\begin{array}{c}\text { Instituto } \\
\text { Nacional de } \\
\text { Asociativismo } \\
\text { y Economía } \\
\text { Social INAES }\end{array}$ & $\begin{array}{l}\text { - Programa «Asistencia a la actividad cooperativa y mutual»: } \\
\text { desarrolla actividades complementarias vinculadas con la promo- } \\
\text { ción y fomento de la actividad cooperativa y mutual y con capaci- } \\
\text { tación en la materia y el registro. Capacita y asiste técnicamente a } \\
\text { cooperativas y mutuales, en particular cooperativas de trabajo con } \\
\text { asociados en condiciones de alta vulnerabilidad social y cooperati- } \\
\text { vas incluidas en programas impulsados por el Estado. Brinda asis- } \\
\text { tencia y capacitación a grupos pre-cooperativos Asiste financiera- } \\
\text { mente a cooperativas. Impulsa un plan anual de fiscalización en } \\
\text { todo el territorio. }\end{array}$ \\
\hline
\end{tabular}




\begin{tabular}{|c|c|}
\hline $\begin{array}{l}\text { Instituciones } \\
\text { responsables }\end{array}$ & Categorías programáticas \\
\hline \multirow[b]{3}{*}{$\begin{array}{c}\text { Ministerio de } \\
\text { Desarrollo } \\
\text { Social }\end{array}$} & Promoción de empleo social \\
\hline & $\begin{array}{l}\text { - Programa nacional de Inclusión socioproductiva y desarrollo local } \\
\left.\text { «Potenciar Trabajo» (Resolución n. }{ }^{\circ} 121 / 2020\right) \text { : tiene el propósito de } \\
\text { mejorar el empleo y generar nuevas propuestas productivas a través } \\
\text { del desarrollo de proyectos socio-productivos, socio-comunitarios, } \\
\text { socio-laborales y la terminalidad educativa. Incluye los beneficia- } \\
\text { rios de programas anteriores como Argentina Trabaja, Ellas Hacen, } \\
\text { Hacemos Futuro y Proyectos Productivos Comunitarios. Su objetivo } \\
\text { es vincular sectores con capacidad para reactivar la producción en } \\
\text { cinco ámbitos considerados prioritarios: construcción, producción de } \\
\text { alimentos, textil, economía del cuidado y recolección y reciclado de } \\
\text { residuos urbanos. Las actividades que se realizan en este programa } \\
\text { brindan como prestación económica individual un salario social } \\
\text { complementario que incluye como una de las posibilidades de con- } \\
\text { traprestación las tareas de cuidado que se realicen dentro del com- } \\
\text { ponente de proyectos socio-comunitarios. Las personas que parti- } \\
\text { cipan deben realizar actividades durante un promedio de } 80 \text { horas } \\
\text { mensuales y acreditar su cumplimiento. }\end{array}$ \\
\hline & $\begin{array}{l}\text { - Monotributo Social Ley } 25.865 \text { : permite la formalización de acti- } \\
\text { vidades económicas, el acceso a las prestaciones de las obras socia- } \\
\text { les y el ingreso al sistema previsional. Para acceder a la cobertura de } \\
\text { una obra social requiere el pago del } 50 \% \text {; el resto del aporte lo cu- } \\
\text { bre el Estado. Una vez registrado como monotributista social, el titu- } \\
\text { lar está en condiciones de emitir facturas por sus productos o servi- } \\
\text { cios y puede convertirse en proveedor del Estado. } \\
\text { — Economía Social: Proyectos «Manos a la Obra» Res. n. }{ }^{\circ} \text { 1375/04: } \\
\text { se otorgan subsidios no reintegrables a los emprendimientos so- } \\
\text { cioproductivos para la adquisición de maquinarias, equipamiento, } \\
\text { herramientas e insumos que promuevan el desarrollo sus activida- } \\
\text { des. Se brinda acompañamiento a proyectos que se encuentran en } \\
\text { un estadio inicial y a los que se encuentran en un proceso de conso- } \\
\text { lidación. En } 2017 \text { se brinda apoyo a } 7.764 \text { destinatarios y } 34 \text { organi- } \\
\text { zaciones (asociaciones de pequeños productores, empresas recupe- } \\
\text { radas, mutuales, cooperativas, ONG que cuentan con proyectos que } \\
\text { se estén iniciando o en vías de consolidación). } \\
\text { - Microcrédito Ley } 26.117: \text { se destina a personas que se organizan } \\
\text { en torno al autoempleo urbano o rural para adquirir insumos y ma- } \\
\text { quinarias que permiten aumentar la productividad y mejorar los in- } \\
\text { gresos familiares. Las organizaciones sociales se constituyen como } \\
\text { instituciones de microcrédito para evaluar, otorgar y realizar un se- } \\
\text { guimiento de los créditos. }\end{array}$ \\
\hline
\end{tabular}




\begin{tabular}{|c|c|}
\hline $\begin{array}{l}\text { Instituciones } \\
\text { responsables }\end{array}$ & Categorías programáticas \\
\hline & $\begin{array}{l}\text { - Marca Colectiva Ley 26.355: distingue los productos y/o servicios } \\
\text { elaborados o prestados por las formas asociativas destinadas al de- } \\
\text { sarrollo de la economía social. Identifica bienes y servicios realizados } \\
\text { por emprendedores para mejorar la calidad de los productos y favo- } \\
\text { recer su comercialización. Requiere estar inscripto en el registro na- } \\
\text { cional de efectores de desarrollo local y economía social. }\end{array}$ \\
\hline $\begin{array}{l}\text { Ministerio de } \\
\text { Desarrollo } \\
\text { Social } \\
\text { (cont.) }\end{array}$ & $\begin{array}{l}\text { - Registro Nacional de Trabajadores y Trabajadoras de la Eco- } \\
\text { nomía Popular (ReNaTEP) Resolución 408/20: se propone reco- } \\
\text { nocer, formalizar y garantizar los derechos de los trabajadores de } \\
\text { la economía popular para que accedan a herramientas que permi- } \\
\text { tan potenciar su trabajo. Quienes se inscriben pueden trabajar de } \\
\text { manera individual o colectiva y en este último caso, las unidades } \\
\text { productivas pueden adoptar, entre otras, la forma de cooperativas } \\
\text { (incluye cooperativas de liberados y empresas recuperadas), proyec- } \\
\text { tos productivos o de servicios (inscriptos en el Registro Nacional de } \\
\text { Efectores Sociales del MDSN), emprendimientos productivos familia- } \\
\text { res y no familiares, organizaciones sociales/comunitarias y núcleos } \\
\text { de agricultura familiar. La incorporación al registro les permite ac- } \\
\text { ceder a programas de trabajo, seguridad social y capacitación, par- } \\
\text { ticipar en redes de comercialización y tener acceso a herramientas } \\
\text { crediticias y de inclusión financiera. El registro se incluyó en la Ley } \\
\text { de Emergencia Social sancionada en diciembre de } 2016 \text { aunque se } \\
\text { habilitó en junio de } 2020 \text {. Los inscriptos en el ReNaTEP trabajan en } \\
\text { su mayoría de forma individual. De más de dos millones de personas } \\
\text { inscritas en mayo de } 2021 \text { solo } 8,4 \% \text { trabaja en cooperativas (Rena- } \\
\text { tep, 2021). }\end{array}$ \\
\hline $\begin{array}{c}\text { Ministerio } \\
\text { de Trabajo, } \\
\text { Empleo y } \\
\text { Seguridad } \\
\text { Social }\end{array}$ & $\begin{array}{l}\text { - Programa Trabajo Autogestionado; competitividad para Em- } \\
\text { presas Autogestionadas. Resolución n. }{ }^{\circ} 203 / 2004 \text {. } \\
\text { - Programa de transición al salario social complementario. Reso- } \\
\text { lución 201-E/2017. } \\
\text { - Programa de Empleo Independiente y Entramados Productivos } \\
\text { locales Resolución } 312 / 20 \text {. Línea de Desarrollo de Entramados } \\
\text { Productivos Locales. }\end{array}$ \\
\hline
\end{tabular}

Fuente: elaborado con base en informaciones sobre los servicios de todos los ministerios, abril 2021.

En forma paralela al programa específico de asistencia a la actividad cooperativa, la primera instancia nacional de institucionalización de políticas dirigidas a la economía social y solidaria fue formalizada en 2004 por el Plan Nacional de Desarrollo Local y Economía Social «Manos a 
la Obra», con el propósito de mejorar la calidad de vida de las familias y generar condiciones favorables para la integración social a través del sistema productivo. En el contexto de las acciones de promoción de empleo social se impulsó en 2009 el Programa de Ingreso Social con Trabajo con el objetivo de crear 100.000 nuevos puestos de trabajo a través de la constitución de cooperativas para la ejecución de obras de baja y mediana complejidad en mejora de la infraestructura local.

Desde la primera década de 2000 los programas implementados por el MDS mantuvieron continuidad y permitieron asegurar y ampliar la cobertura, mejorando las asignaciones presupuestarias ${ }^{11}$. En conjunto implicaron un avance primordial con relación a los programas de transferencias condicionadas de ingresos que los precedieron y respecto de cualquier otro programa social ${ }^{12}$. En su desarrollo durante más de una década, sin dejar de sostener las inversiones requeridas ampliaron la incorporación de los entes ejecutores ${ }^{13}$ (aún en gobiernos de distinto signo político) e incorporaron cambios en su orientación particularmente entre los años 2015-2019 en que se sustituyó el requisito de la prestación laboral por el logro de la terminalidad educativa y la capacitación, en búsqueda de la progresiva autonomía económica de las personas a título individual más que en términos del estímulo a la conformación de iniciativas colectivas de autogestión. El balance de los resultados en distintas coyunturas ha puesto de manifiesto un conjunto de limitaciones, algunas relativas a la masificación de los programas, y el impacto dispar en la consolidación y perdurabilidad de numerosas experiencias

11 Cabe destacar la importancia que reviste la incorporación en la legislación nacional de un conjunto de normas que respondieron a la necesidad de consolidar y formalizar las actividades de los grupos conformados bajo la figura de cooperativa de trabajo. En este caso se destaca la importancia de las leyes de Monotributo Social, de Promoción del Microcrédito y de Marcas Colectivas.

12 Distintos estudios han caracterizado los alcances del programa aunque también mencionaron sus límites para consolidar y resolver la problemática del trabajo no registrado y la inserción en el mercado de trabajo (Arcidiácono y Bermúdez, 2015; Zarazaga, 2015, Vuotto, 2012).

13 Los entes ejecutores están conformados por gobiernos provinciales, municipales, organismos públicos, ministerios etc. También pueden participar como entes ejecutores las cooperativas y otras organizaciones de la sociedad civil. Son responsables de coordinar la ejecución de los programas Argentina Trabaja y Ellas Hacen en distintos espacios geográficos. El plan de actividades se encuentra definido por el MDS. En 2016 se incorporaron más de sesenta entes ejecutores, en su mayoría representando organizaciones sociales y se realizaron acuerdos para el manejo de los fondos para la compra de herramientas e insumos del plan Argentina Trabaja. La Confederación de Trabajadores de la Economía Popular (ver Amova y Vuotto, 2019) tuvo a su cargo veinte entes ejecutores en el conurbano bonaerense. 
Desde 2020, el Programa Potenciar Trabajo en el ámbito del MDS ha incluido los programas Hacemos Futuro ${ }^{14}$ y Salario Social Complementario en un único plan. Su objetivo consiste en contribuir al mejoramiento de la empleabilidad y la generación de nuevas propuestas productivas, a través de la terminalidad educativa, la formación laboral y la certificación de competencias. También contempla la creación y el fortalecimiento de unidades productivas para promover la inclusión social plena y el incremento progresivo de ingresos para alcanzar la autonomía económica. Para mitigar las consecuencias de la crisis económica, desde fines de 2020 se ha incrementado el presupuesto de los programas Potenciar Trabajo y el Potenciar Joven con la finalidad de sustituir el Ingreso Familiar de Emergencia ${ }^{15}$. Asimismo, se mantiene desde 2016 la continuidad del Programa Nacional de Economía Social16 para acompañar a las provincias en sus proyectos socioproductivos reconociendo su contribución como herramienta federal de promoción social y de generación de trabajo.

Junto con las políticas anteriores en el ámbito nacional el MTEySS implementa el Programa Trabajo Autogestionado reglamentado por la Secretaría de Empleo con el objetivo es mantener y generar puestos de trabajo, promocionando y fortaleciendo unidades productivas autogestionadas y mejorar su competitividad y sustentabilidad, así como las condiciones de higiene y seguridad de los trabajadores, promoviendo la mejora de las condiciones y el medio ambiente de trabajo. Se orienta específicamente a las iniciativas de trabajo autogestionado para brindar apoyo técnico y económico en especial a empresas o fábricas recuperadas por sus trabajadores, que se encuentren en funcionamiento o en proceso de reactivación. En función de la experiencia desarrollada y con la finalidad de fortalecer una herramienta de la política pública de empleo, el MTySS reformuló aspectos vinculados con las condicio-

14 El 8 de febrero de 2018, los distintos programas del PRIST se unificaron bajo el programa "Hacemos Futuro» (Resolución n. ${ }^{\circ}$ 96/2018).

15 El Ingreso Familiar de Emergencia fue una medida excepcional implementada por el gobierno nacional para proteger a las familias ante la pérdida o disminución de sus ingresos por la situación de emergencia sanitaria generada por el COVID-19. Constituyó una prestación económica que alcanzó cerca de 9 millones de trabajadores de la economía informal y otras categorías que se encontraban desempleadas.

16 El Plan «Creer y Crear» (Res. n. ${ }^{\circ} 457 / 2016$ y n. $\left.{ }^{\circ} 123 / 2016\right)$ prosiguió el desarrollo de las actividades promovidas desde 2006 e incluyó otras vinculadas con la Responsabilidad Social orientándose a las poblaciones en situación de vulnerabilidad social e impulsando salidas alejadas de la generación de trabajo autogestionado y de la promoción de organizaciones colectiva para reforzar una salida individual. 
nes de acceso y permanencia en el programa en abril de 2017 (Res. n. ${ }^{\circ}$ 62/E (2017).

Las políticas gubernamentales implementadas en el campo del cooperativismo, en particular desde 2003, han sido diversas y continúan manteniendo especificidad. En el curso de más de un siglo de desarrollo del movimiento se transitó de una escasa consideración, en algunas coyunturas, al reconocimiento sobre su importancia y sus demandas a fin de dar respuesta a algunas de sus reivindicaciones. Esto le permitió al Estado asumir una posición activa, al poner en marcha iniciativas de distinto tipo que incluyeron mayoritariamente, desde inicios del siglo XXI a las cooperativas y desde 2016 a las iniciativas de la economía popular, algunas de cuyas expresiones se concretan a través de cooperativas de trabajo.

Un balance de las políticas de estímulo y promoción al cooperativismo durante cerca de dos décadas pone de manifiesto la significación de los instrumentos implementados en torno al cooperativismo de trabajo. Al respecto es importante subrayar que este sector solo puede contribuir a la solución de los problemas de empleo e inserción, en la medida que las políticas que lo promueven se vinculan con estrategias regionales de desarrollo. Los programas de estímulo y promoción al cooperativismo, en sí mismos, no constituyen más que una parte de las opciones de política de inclusión social (Levitas et al., 2007; Hills, 1999) y no siempre garantizan un sendero de inclusión para los sectores relegados.

\section{Conclusiones}

La posición del cooperativismo argentino —-delineada precedentemente a grandes rasgos - y los desafíos provenientes del contexto permiten identificar de manera simplificada tres campos organizacionales:

El primero está conformado mayoritariamente, desde su origen, en torno a entidades de productores entre las que sobresalen las del sector agrario. Algunas de estas cooperativas, que alcanzaron su madurez antes de la década de $1990^{17}$, han profundizado en formas específicas de cooperación y avanzaron en la implementación de políticas de manejo sostenible de los agroecosistemas, en un entorno que se ha vuelto cada vez más competitivo. Coexisten en este conjunto, varias entidades

17 De las cooperativas vigentes registradas en el INAES en 2021, un total de 1829 son entidades creadas entre 1907 y 1990. 
en situación vulnerable. En general dependen de producciones intensivas tradicionales o nuevas, que se destinan al mercado interno, alcanzan bajos rendimientos e ingresos y sus productores no tienen acceso a fuentes de financiamiento suficientes para alcanzar un mejor desempeño.

El segundo conjunto está integrado por las cooperativas de trabajadores, en particular las fundadas a partir de 1990 con el propósito de responder a los grandes desafíos del siglo xxI: desocupación, pobreza y equidad social. En un mercado de trabajo con más de 9,566 millones de personas asalariadas registradas, la informalidad configura uno de los problemas más notorios en las últimas décadas. En este escenario, los sectores más precarizados del empleo han sido afectados con particular gravedad por la pandemia ${ }^{18}$. Para responder a los desafíos, distintos movimientos sociales han apoyado la conformación de cooperativas que contemplan la creciente precarización con el propósito de generar oportunidades de inserción y estabilidad. En un conjunto heterogéneo en el que actúan las cooperativas surgidas de empresas recuperadas, con las cooperativas creadas a partir de la implementación de planes sociales, y las iniciativas propias de los movimientos de la economía popular, conviven lógicas diferentes según el origen y el tipo de demandas y necesidades. Las problemáticas más importantes se estructuran en torno a los derechos al trabajo. Por un lado, el reconocimiento de la condición de trabajador en particular para quienes se encuentran en situaciones de precariedad y por otro el acceso a condiciones que aseguren igual protección que la establecida para los trabajadores en relación de dependencia de la actividad.

En el tercer conjunto, integrado por cooperativas proveedoras de servicios públicos esenciales, se destaca un $60 \%$ de las entidades que brindan el servicio de electricidad en quince provincias del país. Conformadas en su origen por los propios usuarios de los servicios, progresivamente se convirtieron en cooperativas multiservicios para configurar la herramienta asociativa de las pequeñas localidades del interior y garantizar a sus poblaciones mejor calidad de vida y preservación del hábitat.

18 El crecimiento de la informalidad como resultante de sucesivas crisis económicas ha sido determinante desde los años 80. Se acentuó en 1987 con el fracaso del plan Austral, en 1989 debido a la hiperinflación y luego con la crisis del Tequila en 1994, la crisis de 2001, la devaluación de 2014, la crisis de 2016 y la devaluación de 2018 para conducir en 2021 a un universo de más de cuatro millones de personas en un mercado de trabajo de 8,7 millones de asalariados formales, entre privados y públicos. 
Por último, en el plano de las intervenciones impulsadas por el Estado a través de las políticas mencionadas se han tratado de poner de manifiesto las acciones de un gran número de instancias y estructuras mediadoras y la multiplicidad de actores institucionales interesados en promover en particular la reinserción laboral de las personas a través de la fórmula cooperativa. Al riesgo de generar una fuerte dependencia entre esas organizaciones y el Estado, lo acompaña la paradoja de consolidar una lógica de planes sociales, cuando la intención manifiesta del movimiento cooperativo y de las organizaciones sociales consiste en revertirla. No siempre el marco institucional ha resultado el más propicio para la implementación de algunos programas. Por ello, las políticas orientadas al conjunto del sector y los enfoques para valorizar la alternativa cooperativa desde la esfera pública - vinculándola con el desarrollo socio-productivo- pueden resultar eficaces para promover la institucionalidad, de manera directa o indirecta, en la medida que aseguren una efectiva participación de los actores concernidos, favorezcan la inclusión económica y social de los más vulnerables y ayuden a afirmar la sostenibilidad de las organizaciones.

\section{Referencias}

Amova, M. y Vuotto, M.: Hacia una representación sindical de la economía popular en Argentina. UN Inter-Agency Task Force on Social and Solidarity Economy (UNTFSSE). Genève, 2019.

ArCidiÁCONo, P. y Bermúdez, Á.: «Clivajes, tensiones y dinámicas del cooperativismo de trabajo bajo programas sociales. El boom de las cooperativas del Programa Ingreso Social con Trabajo - Argentina Trabaja». Revista del Centro de Estudios de Sociología del Trabajo n. ${ }^{\circ}$ 7, 2015, pp 3-36. Recuperado de https://ojs.econ.uba.ar/index.php/CESOT/article/view/1437

BÓREA, D.: La mutualidad y el cooperativismo en la República Argentina. Tercer Censo Nacional levantado el $1 .^{\circ}$ de junio de 1914. Tomo X. Valores mobiliarios y estadísticas diversas. Recuperado de http://www.deie.mendoza.gov. ar/backend/uploads/files/2016-09-15\%2018:57:06_1914\%20tomo\%20 10\%20-\%201ra\%20parte.pdf

CAletti, A.: El marco histórico del país y el cooperativismo, Cuadernos de Economía Social, 28, Intercoop: Buenos Aires, 1988, pp. 24-31.

CEPAL COOPERATIVISMO LATINOAMERICANO: antecedentes y perspectivas, Libros de la CEPAL n. ${ }^{\circ}$ 14. Santiago de Chile, 1989. Recuperado de https://repositorio. cepal.org/handle/11362/2097

CoOperativa Obrera: Balance social cooperativo. Ejercicio 108. Bahía Blanca 2019. Recuperado de https://www.cooperativaobrera.coop//p/balancesocial-cooperativo-108/ 
CORTÉs CONDE, R.: Problemas del crecimiento industrial de la Argentina (18701914)», Desarrollo Económico, 3. 1963 Recuperado de https://www.jstor. org/stable/3465953?seq=1

CracognA, D.: Manual de Legislación Cooperativa, Buenos Aires: Intercoop. 2016.

Di Tella, T.; Germani, G.; Graciarena, J.: Argentina Sociedad de masas, Buenos Aires, 1965, pp. 284.

Ferrer, A.: La economía argentina, las etapas de su desarrollo y problemas actuales, Fondo de Cultura Económica, Buenos Aires, 1968.

Germani, G.: Política y sociedad en una época de transición. De la sociedad tradicional a la sociedad de masas, Editorial Paidós: Buenos Aires, 1962.

HILls, J.; LE GRAND, J.; PIACHAUd, D. (eds.): Understanding social exclusion, Oxford University Press: 2002.

INAES: Registro Nacional de Empresas Recuperadas, Buenos Aires, 2021, en https://www.argentina.gob.ar/inaes/registro-nacional-de-empresas-recuperadas

INACYM: Reempadronamiento Nacional y Censo Económico Sectorial de Cooperativas y Mutuales. Buenos Aires: 2008.

Kaplan de Drimer, A.; Drimer, B.: Las cooperativas. Fundamentos-Historia-Doctrina Intercoop, Buenos Aires: 2017, pp. 511-544.

KENDALL, J.: The third sector and the development of European public policy: Frameworks for analysis?, Civil Society, Working Paper 19, London School of Economics and Political Science, July 2001.Recuperado de https://socialpolicy.ucc.ie/Kendall_Third_sector.pdf

LeVIn, A.; VerbeKE, G.: El cooperativismo argentino en cifras, tendencias en su evolución: 1927-1997. Documento del Centro de Estudios de Sociología del Trabajo, n. ${ }^{\circ}$ 6, diciembre de 1997. Recuperado de http://bibliotecadigital. econ.uba.ar/econ/collection/docin/document/docin_cesot_006

Levitas, R.; Pantazis, C.; Fahmy, E.; Gordon, D.; Lloyd-Reichling, E.; Patsios, D., Department of Sociology and School for Social Policy Townsend Centre for the International Study of Poverty, University of Bristol 2007. Recuperado de https://www.researchgate.net/publication/267222796_The_MultiDimensional_Analysis_of_Social_Exclusion

Pedro, B.; Campari, G.; Di Virgilio, M.; Rodríguez, M.; Zapata, M.C.: Construcción autogestionaria de hábitat por cooperativas: derecho a la ciudad y a la centralidad en la Ciudad de Buenos Aires, Ley n. 341/00. Ciudad Autónoma de Buenos Aires: Universidad de Buenos Aires. Secretaría de Ciencia y Técnica, 2020. Recuperado de http://repositoriouba.sisbi.uba.ar/ gsdl/collect/libuba/index/assoc/HWA_3212.dir/3212.PDF

VuotTo, M.: Organizational dynamics of worker cooperatives in Argentina. Service Business. Collection Business and Economics. Volume 5 / 2012. Recuperado de https://doi.org/10.1007/s11628-011-0128-4

Vuotto, M.; Estrago, A.: A propósito de indicadores y estadísticas sobre el cooperativismo argentino, Cuadernos de Cultura Cooperativa 86, 2021.

Vuotro, M.: El cooperativismo de trabajo en la Argentina: contribuciones para el diálogo social, Buenos Aires, OIT Documento 217, 2011. Recuperado 
de https://www.ilo.org/wcmsp5/groups/public/---americas/---ro-lima/documents/publication/wcms_179395.pdf

ZARAZAGA, R.: Los programas de transferencias monetarias condicionadas en Argentina: Análisis sobre el alcance, las condicionalidades y el clientelismo en la Asignación Universal por Hijo y el Programa Argentina Trabaja, Desarrollo Económico, Vol. 54, n. ${ }^{\circ} 214$, pp. 333-356, 2015. Recuperado de https://www.jstor.org/stable/43748264?seq=1 


\section{Derechos de autor}

La revista Deusto Estudios Cooperativos es una revista de acceso abierto lo que significa que es de libre acceso en su integridad inmediatamente después de la publicación de cada número. Se permite su lectura, la búsqueda, descarga, distribución y reutilización legal en cualquier tipo de soporte sólo para fines no comerciales y según lo previsto por la ley; sin la previa autorización de la Editorial (Universidad de Deusto) o el autor, siempre que la obra original sea debidamente citada (número, año, páginas y DOI si procede) y cualquier cambio en el original esté claramente indicado.

\section{Copyright}

The Deusto Journal of Cooperative Studies is an Open Access journal which means that it is free for full and immediate access, reading, search, download, distribution, and lawful reuse in any medium only for non-commercial purposes, without prior permission from the Publisher or the author; provided the original work is properly cited and any changes to the original are clearly indicated. 